\title{
Viscoelastic response of apple flesh in a wide range of mechanical loading rates
}

\author{
Zbigniew Stropek* and Krzysztof Golacki \\ Department of Mechanical Engineering and Automatic Control, University of Life Sciences in Lublin, \\ Głęboka 28, 20-612 Lublin, Poland
}

Received September 18, 2017; accepted February 20, 2018

\begin{abstract}
The cylindrical samples of 'Beni Shogun' apples cultivar in a range of deformation velocities from 0.0002 to $1 \mathrm{~m} \mathrm{~s}^{-1}$ were studied using stress relaxation tests. In the work, experimental courses of the force response were described via the Maxwell model, and the effects of deformation velocity on the Maxwell model parameters as well as the maximum and residual force were determined. The maximum force increased with the increase of the deformation velocity, which proved the response of apple flesh to be of viscoelastic nature. The residual force described the state of the material after the strain and was much higher under the quasi-static than impact loading conditions. The three relaxation times decreased with the increasing deformation velocity. For the shortest relaxation time (order of magnitude $0.1 \mathrm{~s}$ ) there was a rapid decrease in the velocities under the quasi-static loading conditions and it remained on a steady and low level under the impact loading conditions. A definite limit was observed between the medium relaxation time (order of magnitude $1 \mathrm{~s}$ ) for the lowest deformation velocity of $0.0002 \mathrm{~m} \mathrm{~s}^{-1}$ and the other relaxation times obtained at higher deformation velocities. The values of the longest relaxation time (order of magnitude $100 \mathrm{~s}$ ) were much larger under the quasi-static than the impact loading conditions.

Keywords: apple, relaxation times, quasi-static and impact loading, Maxwell model, stress relaxation
\end{abstract}

\section{INTRODUCTION}

Stress relaxation tests are an essential source of knowledge about the physical state of high water content agricultural products such as fruit and vegetables. Their results are used to determine damage resistance (Markowski et al., 2006; Rajabipour et al., 2004), ripeness extent (Chauhan et al., 2006; Hassan et al., 2005; Ince et al., 2016), differences between the cultivars (Blahovec and Esmir, 2001), and consumer quality (Blahovec, 2003; Markowski and Zielińska,

*Corresponding author e-mail: zbigniew.stropek@up.lublin.pl
2013). The tests are of particular significance because their results make it possible to propose a model of the studied material. The most common of these are applied to describe the rheological properties of the biological material. Such tests are linear, and are presented in the form of a mechanical analog composed of a spring and dashpot system (Del Nobile et al., 2007; Escalante-Aburto et al., 2017; Wang, 2003). One of these is the Maxwell model constituting a series connection of a spring and a dashpot (Khazaei and Mann, 2005; Saeidirad et al., 2013; Szot and Gołacki, 1999; Zhao et al., 2017). In order to improve approximation accuracy of the experimental course, the number of model elements is increased. As it is usually composed from 2 to 3 elements, in such work as ours, 4 to 6 coefficients are thus necessitated.

A number of difficulties accompany the choice of the specified model employed to describe accurately the mechanical behaviour of the plant material under different loading conditions. This is because fruit and vegetables are characterised by natural changeability, viscoelastic response and by the structural form of the cellular tissue. Satisfying these requirements leads to the expansion of models and their complexity. Therefore, the ideal mathematical representation of a given physical phenomenon should include the lowest possible number of coefficients with their reasonable physical interpretation. The mathematical form of the model should also be characterised by simplicity and be susceptible to changes of physical parameters and not 
be susceptible to those of random experimental parameters. Therefore, the 6-parameter Maxwell model was used in the research.

Models based on the theory of linear viscoelasticity describe the mechanical response of fruit and vegetables on the assumption that the material deforms in a certain steady way. However, the cellular tissue of biological materials has a structural form and cannot be treated as a physical continuum. Hence, it is interesting to find such parameters which would allow with high accuracy to relate stress and strain states in the macro scale to the internal stress and strain of cellular structure individual elements.

The aim of the study was, firstly, to determine the apple flesh response in a wide range of deformation velocities - from 0.0002 to $1 \mathrm{~m} \mathrm{~s}^{-1}$ - for both the quasi-static and impact loading conditions; secondly, to determine the changeability of the Maxwell model parameters, as well as maximum and residual force in the function of deformation velocity; thirdly, to enhance the interpretation of changes of the Maxwell model parameters and the quantities characterising the experimental course in relation to the apple flesh tissue structure under quasi-static and impact loading conditions.

\section{MATERIALS AND METHODS}

Cylindrical samples of the 'Beni Shogun' cultivar apple $20 \mathrm{~mm}$ in diameter and height were studied. The identical value of diameter and height eliminated the effect of sample sizes on the parameters of the assumed model. Three cylindrical samples were cut from each apple in order to take the stress relaxation test at three deformation velocities. This cutting method was applied under both loading conditions. Its aim was to decrease the measurement results scatter and to determine flesh response of the same apple at different deformation velocities under a given loading condition. The samples were cut by means of a manual punching die with the internal diameter $20 \mathrm{~mm}$ parallel to the vertical apple axis going through the calyx and stem. A reproducible sample height was obtained by cutting out a cylindrical specimen in the case of $20 \mathrm{~mm}$ height.

The samples were initially compressed along the $2 \mathrm{~mm}$ axes and then the steady strain was maintained while recording the force response for $30 \mathrm{~s}$. The assumed test time resulted from small changes of the force response after $30 \mathrm{~s}$. Stress relaxation tests under the quasi-static loading conditions were applied by means of the TA.HD plus Texture Analyser at three velocities: 0.0002, 0.002, $0.02 \mathrm{~m}$ $\mathrm{s}^{-1}$. The measurements under impact loading conditions were made on an impact research stand (Stropek and Gołacki, 2016a) at the velocities of: $0.25,0.5$, and $1 \mathrm{~m} \mathrm{~s}^{-1}$. The sample deformation size amounting to $2 \mathrm{~mm}$ was determined from the initial destructive compression tests taken under quasi-static loading conditions. It was assumed that the sample deformation size corresponds to that of measu- ring head displacement for the force constituting around $60 \%$ of that resulting in sample failure. The tests were applied at $24^{\circ} \mathrm{C}$. For each deformation velocity, 15 replications were made. As the research was performed at six deformation velocities, in total, 90 stress relaxation tests were taken.

Previous research has shown that the viscoelastic properties of fruits and vegetables determined by the Maxwell model parameters are dependent on the texture condition of the material. Hence, flesh firmness measurements were performed by means of the manual Magness-Taylor penetrometer. For each apple, twice repeated measurements were made. The firmness test consisted in determining the maximum force required for punching through the apple flesh with a bar of 7/16 inch diameter to a $8 \mathrm{~mm}$ depth at constant velocity. The apple skin and thin flesh layer were first removed with a special knife. The accuracy of firmness measurements was $1 \mathrm{~N}$. The mean and standard deviation values of 'Beni Shogun' apples flesh firmness at the $24^{\circ} \mathrm{C}$ amounted to $40.4 \pm 3.1$. In total, 30 firmness measurements were made.

The device for cylindrical samples impact measuring consisted of a pendulum with a rigid arm ended with a cylindrical hammer. In this, a piezoelectric force sensor was placed. The hammer mass was adjusted so that kinetic energy, even at low heights, was much bigger than that of sample deformation. Thus, it can be assumed that the sample was being deformed at constant velocity. The pendulum arm length was $940 \mathrm{~mm}$, and the hammer displacement during the contact with a sample amounted to $2 \mathrm{~mm}$. Therefore, it can be assumed that the sample deformation proceeded along a straight line. The anvil in the form of a cylinder was attached to a vertical concrete wall. The cylindrical sample was fixed to the vertical plate screwed in the anvil with the use of technical vaseline. A WMU45SK angle sensor was mounted to the pendulum axis which allowed the measurement of pendulum angular displacement from the perpendicular, and as a result, enabled a determination of drop height and deformation velocity. The force measurement during the impact was made by means of the piezoelectric force sensor, ENDEVCO 2311100 , with the sensitivity $24.23 \mathrm{mV} / \mathrm{N}$ and the measurement range $\pm 220 \mathrm{~N}$. The microprocessor recorder, which next transmitted the data to a computer, collaborated with the force sensor. The sampling frequency of the microprocessor recorder was $2.06 \mathrm{kHz}$. Under the quasi-static loading conditions, the research was carried out on the two-column TA.HD plus Texture Analyser of the Stable Micro Systems company equipped with the load cell of $300 \mathrm{~N}$ capacity. The sampling frequency during the test amounted to $200 \mathrm{~Hz}$. The measurement was triggered when the force response exceeded $0.3 \mathrm{~N}$. 
The 6-parameter Maxwell model was applied to describe the stress relaxation curves of apple flesh. There was used the formula given by Chen and Fridley (1972) for the force response of the sample compressed along the axis:

$$
F(t)=\left(\frac{S}{1} \int_{0}^{1} \int_{m}^{f_{m}}\left(E_{0}+\sum_{i=1}^{2} E_{\mathrm{i}} \mathrm{e}^{-\frac{E_{i}}{\eta_{\mathrm{i}}}\left(f_{m}-f\right)}\right) d t\right) \mathrm{e}^{-\frac{E_{i}}{\eta_{\mathrm{i}}}\left(t+f_{m}\right)}
$$

where: $S$ is the cross section area $\left(\mathrm{m}^{2}\right), v$ is the deformation velocity $\left(\mathrm{m} \mathrm{s}^{-1}\right), l$ is the sample length $(\mathrm{m}), t_{m}$ is the increasing deformation time (s) and $t$ is the time counted from the beginning of sample deformation (s), $e$ is the base of the natural logarithm.

Equation (1) describes the test phase where the constant strain was maintained $\left(t>t_{m}\right)$. It includes the $v$ deformation velocity, which proves that the stress relaxation phenomenon was considered at the time when the sample was deformed. The force response curves obtained from the experiment were approximated by Eq. (2):

$$
F(t)=\sum_{i=1}^{3} A_{i} e^{-\alpha_{\imath}\left(t-t_{m}\right)},
$$

in which $A_{i}$ and $\alpha_{i}$ are the parameters.

We applied the nonlinear minimisation Quasi-Newton method to determine the values of the aforementioned parameters. 33 measuring points were used to approximate the experimental course in the following manner: the first 10 points every $0.01 \mathrm{~s}$, the next 10 every $0.1 \mathrm{~s}$, the successive 9 every $1 \mathrm{~s}$ and the last 4 every $5 \mathrm{~s}$. Through a comparison of Eqs (1) and (2), $E_{i}$ and $\eta_{i}$ model parameters were determined (Chen and Chen, 1986; Stropek et al., 2014). These are described via Eqs (3) and (4):

$$
\begin{aligned}
E_{i} & =\frac{A_{i} l \alpha_{i}}{S \nu\left(1-e^{-\alpha_{i} t_{m}}\right)}, \\
\eta_{i} & =\frac{A_{i} l}{S v\left(1-e^{-\alpha_{i} t_{m}}\right)} .
\end{aligned}
$$

The results were statistically analysed with Statistica 13. The statistical significance of the differences between the mean values of the studied quantities was determined using a one-way analysis (ANOVA). The least significant difference (LSD) test at a significance level of 0.05 was applied.

\section{RESULTS AND DISCUSSION}

Maximum and residual forces were determined from the experimental courses. The maximum force was recorded at the end of the initial deformation period of time, whereas the residual force at the end of the stress relaxation test. Figure 1 shows the dependence of the maximum and residual forces on the deformation velocity.

The maximum force increased with the increasing deformation velocity. Viscoelastic response of apple flesh during the stress relaxation test indicated that the material was characterised by greater resistance with increasing

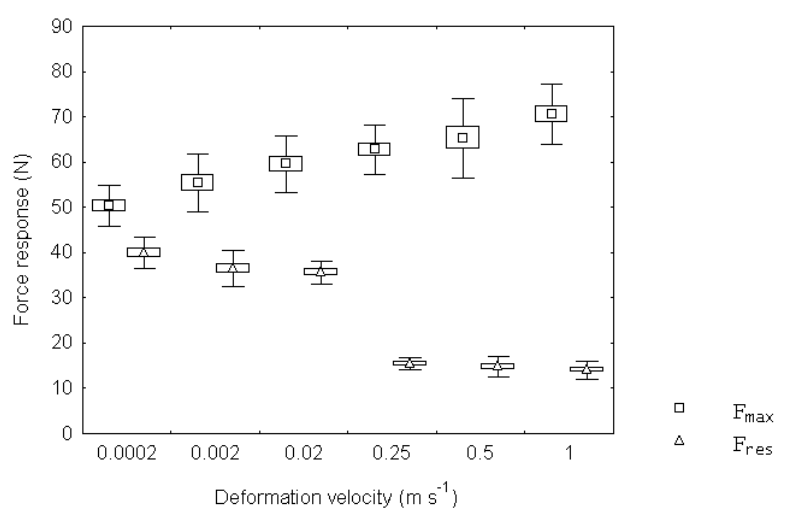

Fig. 1. Dependence of the maximum and residual forces on the deformation velocity.

deformation velocity. This viscoelastic behaviour of biological material was effective only at the time at which the sample deformation increased.

Baritelle and Hyde (2000) studied the damage response of cylindrical samples of two pear cultivars during dynamic, axial compression at the strain rate from 50 to $150 \mathrm{~s}^{-1}$, which for sample length amounting to $15.1 \mathrm{~mm}$, was equivalent with the deformation velocity from 0.75 to $2.25 \mathrm{~m} \mathrm{~s}^{-1}$. They found that the failure stress increased with the increasing strain rate, whereas the failure strain remained almost the same. Thus, the tissue toughness and stiffness increased with the increasing strain rate. An increase of the peak force response and maximum acceleration with the increase of drop height under the impact loading conditions was also observed in apples (Lu and Wang, 2007; Stropek and Gołacki, 2013), peaches (Brusewitz et al., 1991; Maness et al., 1992; Zhang and Brusewitz, 1991) and kiwi fruits (Mc Glone et al., 1997). The residual force was recorded at the end of the experiment, therefore, it described the material condition after the strain. The residual force decreased with the increasing deformation velocity. The differences between the mean values of the residual force under the quasi-static loading conditions were statistically significant in comparison with those under the impact loading conditions. In addition, the values of the residual force under the quasi-static loading conditions were much higher than under the impact loading ones. This implies that a significant increase of tissue damage occurred in the samples of the apple flesh with the increasing deformation velocity.

Peleg and Calzada (1976) found that the residual force at a rate of $0.0017 \mathrm{~m} \mathrm{~s}^{-1}$ was lower than at a rate of $0.00017 \mathrm{~m}$ $\mathrm{s}^{-1}$ for cylindrical samples of apples, pears and potatoes. This situation was observed in the case of deformation larger than $5 \%$ for apples and pears and $10 \%$ for potatoes, and can account for irreversible changes due to biological material strain. The other parameters confirming plastic deformations during the load are: the permanent deformation and plastic deformation energy. In the case of apples, 


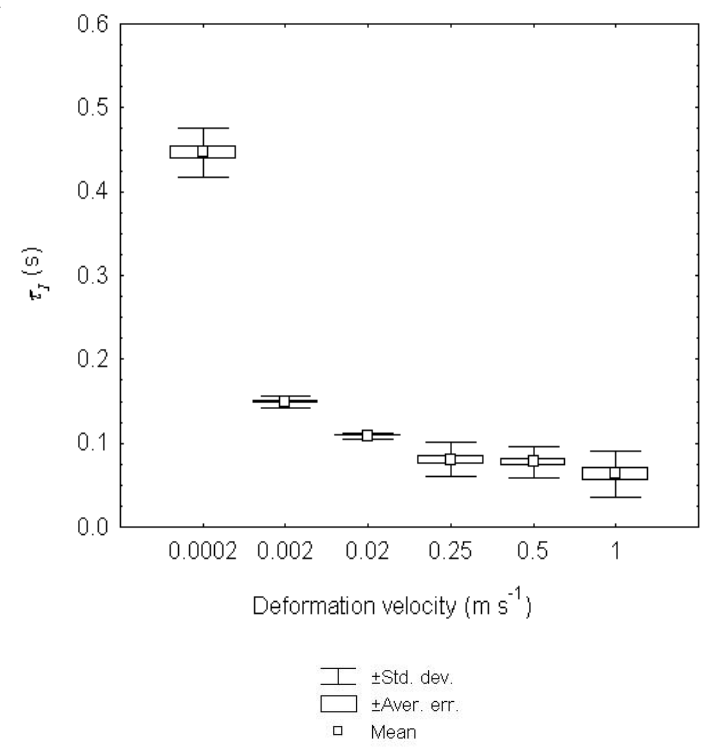

b
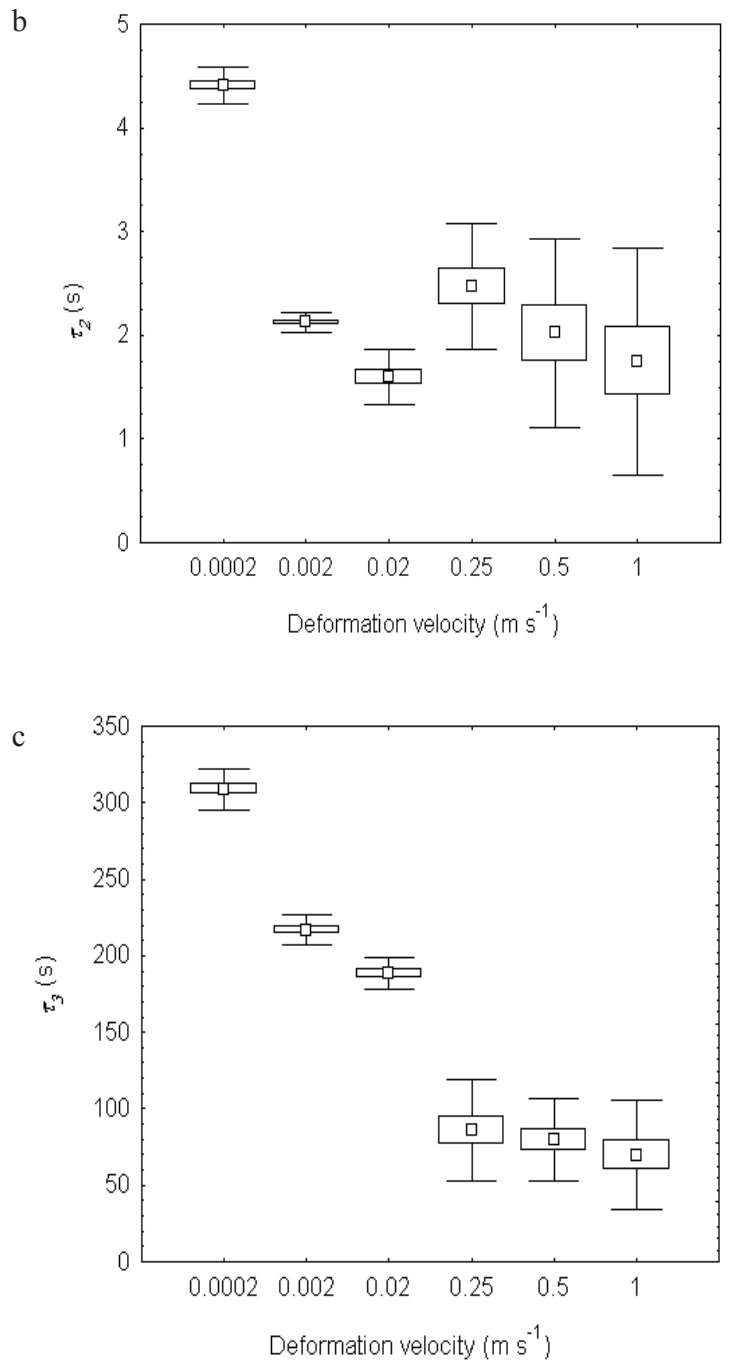

Fig. 2. Relationship between the: $\mathrm{a}-\tau_{1}, \mathrm{~b}-\tau_{2}, \mathrm{c}-\tau_{3}$ relaxation time and the deformation velocity. under impact loading conditions, permanent deformation ( $\mathrm{Lu}$ and Wang, 2007; Stropek and Gołacki, 2015) and plastic deformation energy (Stropek and Gołacki, 2016b) increased with the increasing impact velocity.

In order to better describe the response of apple flesh to the load, three new parameters, the $\tau$ 'relaxation times', were applied. These are the quotients of $\eta_{1} E_{1}^{-1}, \eta_{2} E_{2}^{-1}$ and $\eta_{3} E_{3}^{-1}$ and show the speed of the force response decrease after rapid deformation. Figure 2 present the relationships between the relaxation times of the Maxwell model individual elements and the deformation velocity.

The $\tau_{1}$ relaxation time decreased with the increasing deformation velocity (Fig. 2a). In the range of deformation velocities corresponding to the quasi-static loading conditions, a rapid decrease of $\tau_{1}$ relaxation time is evident. However, for velocities under impact loading conditions, the $\tau_{1}$ relaxation time stabilised at a low, steady level. The differences between the mean values of the $\tau_{1}$ relaxation time at the velocities under the quasi-static loading conditions were statistically significant, whereas at those under the impact loading conditions, no significance was observed.

The $\tau_{2}$ relaxation time also decreased with the increasing deformation velocity (Fig. 2b). Still, for the $\tau_{2}$ relaxation time, there was a distinct limit between the $\tau_{2}$ value corresponding to the lowest deformation velocity (amounting to $0.0002 \mathrm{~m} \mathrm{~s}^{-1}$ ) and the other $\tau_{2}$ values for higher deformation velocities (from 0.002 to $1 \mathrm{~m} \mathrm{~s}^{-1}$ ) independent of the loading conditions.

The $\tau_{3}$ relaxation time decreased with the increase in deformation velocity (Fig. 2c). The differences between the mean values of the $\tau_{3}$ relaxation time were statistically significant for the deformation velocities in the range of $0.0002-0.02 \mathrm{~m} \mathrm{~s}^{-1}$ (quasi-static conditions), whereas for the velocities in the range of $0.25-1 \mathrm{~m} \mathrm{~s}^{-1}$ (impact conditions), the impact of the deformation velocities on the mean values of the $\tau_{3}$ relaxation time was not found. Figure $2 \mathrm{c}$ shows that the values of the $\tau_{3}$ relaxation time were much larger under the quasi-static than impact loading conditions.

The relaxation times of individual elements of the Maxwell model can be used to interpret the phenomena taking place in the material under loading. Chen (1994) presented the description of pear flesh behaviour under loading due to the creep test. He determined three relaxation times based on the Maxwell model. According to his interpretation, the relaxation time with the smallest value represented the gases which flew in the intercellular spaces at high velocity and were able to support the load for a short time, while the relaxation time with the mean value described the liquids flow in the intercellular spaces. Therefore, liquids can sustain the load for a longer period of time than gases. Thus, the relaxation time with the largest value represents cells as a whole in which the contained liquid relocates at a very slow velocity. 
Based on the above description of relaxation times, it can be stated that in load sustaining, the share of gases and fluids in the intercellular spaces, as well as whole cells, decreased along with the increase of the deformation velocity. Furthermore, the share of gases in the load transfer suddenly decreased under quasi-static loading conditions, but reached a steady, low level under impact loading conditions (Fig. 2a). For the share of liquid in the load sustaining, a definite limit exists between the lowest deformation velocity of $0.0002 \mathrm{~m} \mathrm{~s}^{-1}$ and the other velocities under different loading conditions (Fig. 2b). The share of cells in the load sustaining was much larger under quasi-static than under impact loading conditions. This share decreased with the increase of deformation velocity under quasi-static loading conditions, whereas it maintained a steady, low level under impact loading conditions (Fig. 2c).

Chen and Chen (1986) developed an experimental method to determine the stress relaxation function at different loading rates. Their research showed that the viscoelastic constants (which are the inverse of the relaxation times), increased with the increasing loading rate. The influence of loading rates on the viscoelastic constants was statistically significant, being $1 \%$ significance level in the range of loading rates from 0.0033 to $0.7 \mathrm{~m} \mathrm{~s}^{-1}$.

The other studies showed different physical phenomena dependent on loading conditions. Calzada and Peleg (1978) found that on the basis of stress-strain curves shape, as well as the obtained stress relaxation and compressibility data, two opposite mechanisms responsible for the stress level can be indicated. One of these mechanisms is internal fracture. This reduces the mechanical strength of the deformed samples, while the other is structural compaction. This results in the increase of the samples' strength. Roudot et al. (1991) used a modelling method based on imaging techniques in order to explain the consequences of the mechanical damage on apples during the impact and compression. Their research confirm the existence of two different physical phenomena dependent on the strain rate. Cell destruction takes place mainly during impact, whereas cell displacement occurs during compression. Holt and Schoorl (1982) showed that horticultural products do not fail according to only one failure criterion. Damage occurring as a result of expanding stress state is dependent on the strength limit under different loading conditions. For example, the potato might fail by cleavage, slip or bruising depending on the loading conditions, while bruise damage is most likely in apples, strawberries and ripe pears, and green pears fail by slipping.

External damages of fruit and vegetables are caused by cell failure on the micro scale, although those of different tissue structure can respond differently to external forces. Cells can fail by bursting or crushing. For example, apple tissue contains a lot of intercellular spaces which are estimated to be approximately $25 \%$ of total apple volume in contrast to pears, nectarines and potatoes which have 12 ,
10, and 3\% airspaces, respectively (Baritelle and Hyde, 2001; Harker and Sutherland, 1993). The larger the intercellular spaces are in the tissue, the more damage related to bruising takes place. Such an effect results from the fact that airspaces, being the weakest element of the structure, reduce tissue strength, thus enabling damage in their immediate proximity. The ripeness process also contributes to cell damage because cells are surrounded by larger intercellular spaces.

\section{CONCLUSIONS}

1. Maximum force increases with the increasing deformation velocity. This indicates the viscoelastic response of apple flesh and demonstrates that the material is characterised by greater resistance to increasing deformation velocity.

2. Residual force is much higher under quasi-static than under impact loading conditions. These differences between the values of the residual force under two different loading conditions show that much greater apple flesh tissue damage comes about under impact than under quasistatic loading conditions.

3 . The three relaxation times decrease with the increasing deformation velocity. For the shortest relaxation time (order of magnitude $0.1 \mathrm{~s}$ ), there is a sudden decrease in the quasi-static range of loading velocities and the maintenance of a steady, low level under the impact conditions. There is an evident limit between the medium relaxation time (order of magnitude $1 \mathrm{~s}$ ) for the lowest deformation velocity of $0.0002 \mathrm{~m} \mathrm{~s}^{-1}$ and the other relaxation times obtained at higher velocities. The values of the longest relaxation time (order of magnitude $100 \mathrm{~s}$ ) are much larger under the quasistatic than impact loading conditions.

4. The research shows that the different behaviour of apple flesh under quasi-static and under impact loading conditions is due to irreversible phenomena occurring in the tissue that is related to pumping over and filtration of the cellular sap, the bursting of cell walls, as well as structure delamination.

Conflict of interest: The Authors declare they have no conflict of interest.

\section{REFERENCES}

Baritelle A.L. and Hyde G.M., 2000. Strain rate and size effects on pear tissue failure. Trans. ASAE, 43, 95-98.

Baritelle A.L. and Hyde G.M., 2001. Commodity conditioning to reduce impact bruising. Postharvest Biol. Technol., 21, 331-339.

Blahovec J., 2003. Activation volume from stress relaxation curves in raw and cooked potato. Int. J. Food Prop., 6, 183-193.

Blahovec J. and Esmir A.A., 2001. Stress relaxation in cooked potato tubers expressed by improved rate controlled model. Int. J. Food Prop., 4, 485-499. 
Brusewitz G.H., McCollum T.G., and Zhang X., 1991. Impact bruise resistance of peaches. Trans. ASAE, 34, 962-965.

Calzada J.F. and Peleg M., 1978. Mechanical interpretation of compressive stress-strain relationships of solid foods. J. Food Sci., 43, 1087-1092.

Chauhan O.P., Raju P.S., Dasgupta D.K., and Bawa A.S., 2006. Instrumental textural changes in banana (var. Pachbale) during ripening under active and passive modified atmosphere. Int. J. Food Prop., 9, 237-253.

Chen P., 1994. Creep response of a generalized Maxwell model. Int. Agrophys., 8, 555-558.

Chen P. and Chen S., 1986. Stress-relaxation functions of apples under high loading rates. Trans. ASAE, 29, 1754-1759.

Chen P. and Fridley R.B., 1972. Analytical method of determining viscoelastic constants of agricultural materials. Trans. ASAE, 15, 1103-1106.

Del Nobile M.A., Chillo S., Mentana A., and Baiano A., 2007. Use of the generalized Maxwell model for describing the stress relaxation behavior of solid-like foods. J. Food Eng., 78, 978-983.

Escalante-Aburto A., Figueroa-Cardenas J.D., Veles-Medina J.J., Ponce-Garcia N., Hernandez-Estrada Z.J., RayasDuarte P., and Simsek S., 2017. Viscoelastic properties of tablets from Osborne fractions, pentosans, flour and bread evaluated by creep tests. Int. Agrophys., 31, 307-315.

Harker F.R. and Sutherland P.W., 1993. Physiological changes associates with fruit ripening and the development of mealy texture during storage of nectarines. Postharvest Biol. Technol., 2, 269-277.

Hassan B.H., Alhamdan A.M., and Elansari A.M., 2005. Stress relaxation of dates at khalal and rutab stages of maturity. J. Food Eng., 66, 439-445.

Holt J.E. and Schoorl D., 1982. Mechanics of failure in fruits and vegetables. J. Texture Stud., 13, 83-97.

Ince A., Cevik M.Y., and Vursavus K.K., 2016. Effect of maturity stages on textural mechanical properties of tomato. Int. J. Agric. Biol. Eng., 9, 200-206.

Khazaei J. and Mann D.D., 2005. Effects of moisture content and number of loadings on force relaxation behaviour of chickpea kernels. Int. Agrophys., 19, 305-313.

Lu L.X. and Wang Z.W., 2007. Dropping bruise fragility and bruise boundary of apple fruit. Trans. ASABE, 50, 1323-1329.

Maness N.O., Brusewitz G.H., and McCollum G., 1992. Impact bruise resistance comparison among peach cultivars. Hort Sci., 27, 1008-1011.

Markowski M., Ratajski A., Konopko H., Zapotoczny P., and Majewska K., 2006. Rheological behavior of hot-airpuffed amaranth seeds. Int. J. Food Prop., 9, 195-203.
Markowski M. and Zielińska M., 2013. Influence of drying temperature and rehydration on selected textural properties of carrots. Int. J. Food Prop., 16, 586-597.

McGlone V.A., Jordan R.B., and Schaare P.N., 1997. Mass and drop-height influence on kiwifruit firmness by impact force. Trans. ASAE, 40, 1421-1428.

Peleg M. and Calzada J.F., 1976. Stress relaxation of deformed fruits and vegetables. J. Food Sci., 41, 1325-1329.

Rajabipour A., Zariefard M.R., Dodd G.T., and Norris E.R., 2004. Tensile strength and relaxation of tomato skin by loop technique. Int. Agrophys., 18, 153-157.

Roudot A.C., Duprat F., and Wenian C., 1991. Modelling the response of apples to loads. J. Agric. Eng. Res., 48, 249-259.

Saeidirad M.H., Rohani A., and Zarifneshat S., 2013. Predictions of viscoelastic behavior of pomegranate using artificial neural network and Maxwell model. Comput. Electron. Agric., 98, 1-7.

Stropek Z. and Golacki K., 2013. The effect of drop height on bruising of selected apple varieties., Postharvest Biol. Technol., 85, 167-172.

Stropek Z. and Golacki K., 2015. A new method for measuring impact related bruises in fruits. Postharvest Biol. Technol., 110, 131-139.

Stropek Z. and Golacki K., 2016a. Methodological aspects of determining apple mechanical properties during impact. Int. J. Food Prop., 19, 1325-1334.

Stropek Z. and Golacki K., 2016b. Quantity assessment of plastic deformation energy under impact loading conditions of selected apple cultivars. Postharvest Biol. Technol., 115, 9-17.

Stropek Z., Gołacki K., Kołodziej P., Gladyszewska B., Samociuk W., and Rejak A., 2014. Effect of polyvinyl alcohol and keratin on stress relaxation course in thermoplastic starch. Przem. Chem., 93, 364-367.

Szot B. and Golacki K., 1999. Influence of moisture content on the stress relaxation response of amaranth seeds. Int. Agrophys., 13, 387-390.

Wang J., 2003. Anisotropic relaxation properties of pear. Biosyst. Eng., 85, 59-65.

Zhang X. and Brusewitz G.H., 1991. Impact force model related to peach firmness. Trans. ASAE, 34, 2094-2098.

Zhao W., Fang Y., Zhang Q., Guo Y., Gao G., and Yi X., 2017. Correlation analysis between chemical or texture attributes and stress relaxation properties of 'Fuji' apple. Postharvest Biol. Technol., 129, 45-51. 